\title{
Lipoxygenation of Arachidonic Acid as a Source of Polymorphonuclear Leukocyte Chemotactic Factors in Synovial Fluid and Tissue in Rheumatoid Arthritis and Spondyloarthritis
}

\author{
Lloyd B. Klickstein, Christine Shapleigh, and Edward J. Goetzl, \\ Howard Hughes Medical Institute Laboratory at Harvard Medical School and \\ Departments of Medicine, Brigham and Women's Hospital and \\ Harvard Medical School, Boston, Massachusetts 02115
}

\begin{abstract}
A B S TRACT The predominant lipoxygenase products of archidonic acid were extracted and purified from synovial fluid and sonicates of synovial tissue of patients with rheumatoid arthritis (RA), spondyloarthritis (SA), or a noninflammatory arthropathy (NIA). The concentration of $5(S), 12(R)$-dihydroxy- $6,8,10$ (trans/trans/cis)-14-cis-eicosatetraenoic acid (leukotriene $B_{4}$ ) in synovial fluid was elevated significantly in patients with RA and a positive latex test for rheumatoid factor $(P<0.05, n=14)$ and in patients with SA $(P<0.05, n=10)$, compared with that of subjects with NIA $(n=9)$. The content of $5(S)$-hydroxy6,8,11,14-eicosatetraenoic acid (5-HETE), but not of leukotriene $B_{4}$, was elevated significantly in synovial tissue of seven patients with RA in comparison with that of four subjects with NIA $(P<0.05)$. A single intra-articular injection of corticosteroid significantly lowered the synovial fluid level of leukotriene $B_{4}$ in six patients with RA. These data suggest an involvement of the potent chemotactic factors 5-HETE and leukotriene $B_{4}$ in human inflammatory disease.
\end{abstract}

\section{INTRODUCTION}

The activation of basophils, mast cells, and polymorphonuclear $(\mathrm{PMN})^{1}$ and mononuclear leukocytes

Address reprint requests to Dr. Edward Goetzl, Seeley Mudd Building, Harvard Medical School, Boston, Mass. 02115.

Received for publication 7 July 1980 and in revised form 12 August 1980.

${ }^{1}$ Abbreviations used in this paper: HETE, mono-hydroxyeicosatetraenoic acid; NIA, noninflammatory arthropathy; PMN, polymorphonuclear; RA, rheumatoid arthritis; SA, spondyloarthritis. by immunological and other stimuli releases arachidonic acid, which is converted in part by lipoxygenation to unstable hydroperoxy-eicosatetraenoic acids. Although hydroperoxy-eicosatetraenoic acids are transformed largely to mono-hydroxy-eicosatetraenoic acids (HETE) (1-4), 5(S)-hydroperoxy-eicosatetraenoic acid is the unique source of a family of complex HETE, termed leukotrienes, that contain additional polar substituents and three conjugated double bonds (5). 5(S)-hydroxy-6(R)-glutathionyl - 7,9-trans - 11,14 - cis - eicosatetraenoic acid (leukotriene $\mathrm{C}_{4}$ ) and 5(S)-hydroxy-6(R)-cysteinylglycine - 7,9-trans - 11,14 - cis - eicosatetraenoic acid (leukotriene $\mathrm{D}_{4}$ ) are functionally critical components of the slow-reacting substance of anaphylaxis $(6,7)$. $5(S), 12(R)$-dihydroxy-6,8,10(trans/trans/cis) - 14-cis eicosatetraenoic acid $\left(5,12\right.$-di-HETE or leukotriene $\left.B_{4}\right)$ and 5(S)-hydroxy-6,8,11,14-eicosatetraenoic acid (5HETE) elicit human neutrophil and eosinophil chemotaxis and chemokinesis, enhance the expression of PMN leukocyte $\mathrm{C} 3 \mathrm{~b}$ receptors, increase the intraleukocyte concentration of guanosine $3^{\prime}: 5^{\prime}$ cyclic monophosphate, and evoke a modest release of lysosomal enzymes from human neutrophils $(3,8-10)$. Although the tissue content of 12-L-HETE has been found to be elevated in the lesions of psoriasis compared with noninvolved epidermis of the same patients (11), the levels of the far more potent chemotactic factors 5-HETE and leukotriene $B_{4}$ have not been assessed previously in human disease states. This report documents significantly higher concentrations of leukotriene $B_{4}$ in peripheral joint synovial fluid of patients with sero-positive rheumatoid arthritis and spondyloarthritis, and a higher content of 5-HETE in synovial tissue in sero-positive 
rheumatoid arthritis than in noninflammatory arthropathies.

\section{METHODS}

[ $\left.{ }^{3} \mathrm{H}\right]-12-\mathrm{L}-\mathrm{HETE}$ was generated by incubating partially purified lipoxygenase from homogenates of $4 \times 10^{10}$ washed human platelets with $2 \mathrm{mCi}$ of $\left[{ }^{3} \mathrm{H}\right]$ arachidonic acid $(52 \mathrm{Ci} /$ mmol, New England Nuclear, Boston, Mass.) in $0.05 \mathrm{M}$ Tris- $\mathrm{HCl} / 0.10 \mathrm{M}$ potassium phosphate buffer $(\mathrm{pH} 8.0)$ containing $20 \mu \mathrm{M}$ indomethacin for $4 \mathrm{~h}$ at $37^{\circ} \mathrm{C}$ as described (12). $\left[{ }^{3} \mathrm{H}\right]-5,12$-di-HETE was generated by incubating $4 \times 10^{8}$ human neutrophils with $2 \mathrm{mCi}$ of $\left[{ }^{3} \mathrm{H}\right]$ arachidonic acid in $10 \mathrm{ml}$ of Hanks' solution containing $0.05 \mathrm{~g}$ of recrystallized ovalbumin $/ 100 \mathrm{ml}, 10 \mu \mathrm{M}$ indomethacin, and 20 $\mu \mathrm{M}$ calcium ionophore $\mathrm{A} 23187$ for $60 \mathrm{~min}$ at $37^{\circ} \mathrm{C}(2,3)$. The radiolabeled HETE were extracted and purified by sequential silicic acid column chromatography and reverse-phase high-performance liquid chromatography as described (2, 8,12 ), and were identified by cochromatography with purified products that had been characterized previously by gas chromatography-mass spectrometry $(2,3,12)$. Human neutrophil chemotaxis was assessed by a modified Boyden chamber technique as described $(3,9)$.

Patient population. Definite and classical rheumatoid arthritis (RA) were defined by the criteria of the American Rheumatism Association and patients were classified as having sero-positive $\mathrm{RA}(\mathrm{RA}+)$ or sero-negative $\mathrm{RA}(\mathrm{RA}-)$ according to the results of a standard latex fixation test for rheumatoid factor. Patients with spondyloarthritis (SA) and peripheral joint arthritis had diagnoses of ankylosing spondylitis $(n=3)$, enteropathic spondylitis $(n=1)$, psoriatic arthritis $(n=1)$, and Reiter's disease $(n=5)$. Patients with a noninflammatory arthropathy such as degenerative or traumatic joint disease served as the control subjects. At the time of collection of the synovial material, three of the patients were taking a total of 75-200 $\mathrm{mg}$ of indomethacin/d, and the others were taking aspirin in some form at a total daily dose of $2,400-6,000 \mathrm{mg}$. Some patients were also receiving hydroxychloroquine, gold, penicillamine, and/or $2.5-15 \mathrm{mg} / \mathrm{d}$ of Prednisone, but none had received intraarticular corticosteroids in either knee joint for 3 mo or more before the collection of synovial samples for the present study.

Extraction, purification, and measurement of the HETE in fluid and tissue samples. Synovial fluid was aspirated from knee joints and centrifuged at $2,000 \mathrm{~g}$ for $10 \mathrm{~min}$ at $4^{\circ} \mathrm{C}$ to remove cells and particulate material; the supernatant fluid was stored at $-70^{\circ} \mathrm{C}$ until the HETE were extracted. 2-g portions of synovial tissue, which had been obtained at surgery and stored at $-20^{\circ} \mathrm{C}$, were minced in $2 \mathrm{ml}$ of Hanks' solution at $4^{\circ} \mathrm{C}$, homogenized for 3 min (Polytron; Brinkmann Instruments, Inc., Westbury, N. Y.), and sonicated for 2 min (Branson Sonic Power Co., Danbury, Conn.; model 140D, $200 \mathrm{~W})$. After $10^{5} \mathrm{dpm}$ of $\left[{ }^{3} \mathrm{H}\right]-12$-L-HETE and $5 \times 10^{4} \mathrm{dpm}$ of $\left[{ }^{3} \mathrm{H}\right]-5,12$-di-HETE were added to each 2-3$\mathrm{ml}$ sample of synovial fluid and synovial tissue sonicate, duplicate 20- $\mu$ l aliquots were removed for protein (13) and DNA (14) assays; the samples were titrated to $\mathrm{pH} 4.0$ with 2 M citric acid. Each sample was extracted three times with $4 \mathrm{ml}$ of chloroform:methanol $(2: 1, \mathrm{vol} / \mathrm{vol})$, the tissue sonicates were extracted three times again with $4 \mathrm{ml}$ of ethyl ether, and the organic phases were pooled and dried under $\mathrm{N}_{2}$.

The HETE were resolved and purified by sequential silicic acid column chromatography and reverse-phase highperformance liquid chromatography on a $4.6 \times 250$-mm Ultrasphere ODS column (Altex Scientific Inc., Berkeley, Calif.) that was equilibrated and developed isocratically with methanol:water:glacial acetic acid (790:210:0.1, vol/vol) at a flow rate of $1 \mathrm{ml} / \mathrm{min}$ as described $(2,8,12)$. The 5,12-di-HETE (leukotriene $B_{4}$ ) and 5-HETE were recognized by comparing retention times with those of standards that had been purified and identified previously by gas chromatography-mass spectrometry $(2,3,5)$. Each product was quantitated by optical density at the wavelength of maximum absorption, using previously established extinction coefficients $(2,5)$. The radioactivity in portions of the 12-L-HETE and 5,12-di-HETE peaks was determined to provide an estimate of recovery.

TABLE I

White Blood Cell Counts and Concentrations of Predominant Lipoxygenase Products in Synovial Fluid of Patients with Arthritis or NIA

\begin{tabular}{rrrrr}
\hline & & \multicolumn{3}{c}{ Synovial fluid } \\
\cline { 3 - 5 } Patient & Diagnosis & 5-HETE & 5,12-di-HETE & Leukocytes \\
\hline & & $n g / m l$ & $n g / m l$ & per m $^{3}$ \\
& & & & \\
1 & NIA & 190 & 31 & $400(25) *$ \\
2 & NIA & 1,330 & 64 & $1,000(0)$ \\
3 & NIA & 355 & 70 & $350(14)$ \\
4 & NIA & 120 & 119 & $400(43)$ \\
5 & NIA & 199 & 38 & $150(0)$ \\
6 & NIA & 249 & 58 & $150(0)$ \\
7 & NIA & 241 & 84 & $600(1)$ \\
8 & NIA & 2,198 & 32 & $100(46)$ \\
9 & NIA & 410 & 35 & $250(28)$ \\
10 & SA & 323 & 407 & $28,500(84)$ \\
11 & SA & 136 & 306 & $10,300(74)$ \\
12 & SA & 41 & 331 & $8,650(68)$ \\
13 & SA & 129 & 229 & $5,900(72)$ \\
14 & SA & 311 & 713 & $30,700(91)$ \\
15 & SA & 209 & 204 & $3,850(88)$ \\
16 & SA & 777 & 67 & $9,400(54)$ \\
17 & SA & 916 & 21 & $8,850(38)$ \\
18 & SA & 689 & 41 & $40,200(91)$ \\
19 & SA & 107 & 44 & $26,800(82)$ \\
20 & RA+ & 345 & 156 & $88,000(80)$ \\
21 & RA+ & 570 & 135 & $12,300(90)$ \\
22 & RA+ & 787 & 458 & $10,200(78)$ \\
23 & RA+ & 616 & 64 & $7,960(81)$ \\
24 & RA+ & 622 & 39 & $34,720(85)$ \\
25 & RA+ & 311 & 62 & $38,500(78)$ \\
26 & RA+ & 596 & 132 & $3,500(69)$ \\
27 & RA+ & 1,067 & 518 & $22,500(74)$ \\
28 & RA+ & 1,556 & 126 & $13,600(83)$ \\
29 & RA+ & 1,275 & 301 & $5,700(49)$ \\
30 & RA+ & 387 & 85 & $14,100(66)$ \\
31 & RA+ & 504 & 88 & $24,800(85)$ \\
32 & RA+ & 86 & 50 & $9,600(65)$ \\
33 & RA+ & 235 & 64 & $16,000(97)$ \\
34 & RA- & 244 & 165 & $430(17)$ \\
35 & RA- & 403 & 17 & $8,050(60)$ \\
36 & RA- & 99 & 52 & $44,800(91)$ \\
37 & RA- & 1,198 & 31 & $1,340(64)$ \\
& & & &
\end{tabular}

* The numbers in parentheses represent the percent PMN leukocytes in each sample. 
The addition of $5 \mathrm{mg}$ of 5-methyl prednisolone acetate to one of each pair of 1-ml samples of synovial fluid from three patients did not alter the recoveries of 5,12-di-HETE or 5-HETE relative to those of the duplicate sample lacking the corticoid. The total nanograms of 5,12-di-HETE and of 5-HETE per milliliter of synovial fluid or per milligram of DNA in synovial tissue were calculated from the quantities recovered and the percent recovery of radiolabeled 5,12 di-HETE and 12-L-HETE, respectively. The reproducibility of the purification procedure was assessed by analyses of a series of replicate portions of several fluids. In each such series, the range of values was $< \pm 15 \%$ of the mean.

\section{RESULTS}

The concentration of 5,12-di-HETE in synovial fluid was significantly higher for patients with RA+ $(P$ $<0.05)$ and patients with SA $(P<0.05)$ than for the control subjects with a noninflammatory arthropathy (NIA) or those with RA- (Table I, Fig. 1). The difference between the concentrations of 5,12-di-HETE in SA and in the NIA control patients was more significant $(P<0.02)$ when the six patients with SA and synovial fluid levels of 5,12-di-HETE above the normal range were analyzed as a separate subgroup. Although the total white blood cell counts and the percentages of PMN leukocytes were higher in the patients with RA or SA than NIA (Table I), no significant correlation was found between the white blood cell count and the 5,12-di-HETE concentration in synovial fluid of patients with RA $+(r=0.13)$ or SA $(r=0.44)$, or of all the patients considered as a group $(r=0.14)$. There was no statistically significant difference between the synovial fluid level of 5-HETE in any of the groups of patients and that of the control subjects. In contrast, the 5-HETE content of synovial tissues was elevated significantly in RA+ patients relative to control subjects with NIA $(P<0.05)$, whereas the 5,12-di-HETE content of the tissues was not increased (Fig. 2). Similar results were obtained when the contents of HETE were expressed in terms of the wet weight of the tissues or the protein concentration of the tissue sonicates.

To assess the relative contribution of the HETE to the overall neutrophil chemotactic activity in inflammatory synovial fluids, the 5,12-di-HETE and 5-HETE were obtained as a mixture by silicic acid chromatography of extracts of 1-ml portions of synovial fluid from four patients with RA+. After determination of the yields of HETE according to recovery of the radioactive tracers, the mixtures of HETE each were resuspended in the appropriate fraction of $1 \mathrm{ml}$ of synovial fluid from a patient with NIA. In dose-response studies using 10-200 $\mu$, the neutrophil chemotactic activity of unextracted portions of synovial fluid from the RA+ patients reached plateau values that ranged from 30.6 to 51.3 net neutrophils/high-power field at a concentration of $50 \mu \mathrm{l} / \mathrm{ml}$ of buffer, whereas $50 \mu \mathrm{l}$ of the synovial fluid from the patient with NIA attracted only 6.9 net neutrophils/high-power field. The increment in neutrophil chemotactic activity in the NIA synovial fluid achieved by the addition of HETE purified from the RA+ synovial fluids permitted an estimation that the HETE accounted for $35-57 \%$ of the chemotactic activity in the corresponding unextracted synovial fluids.

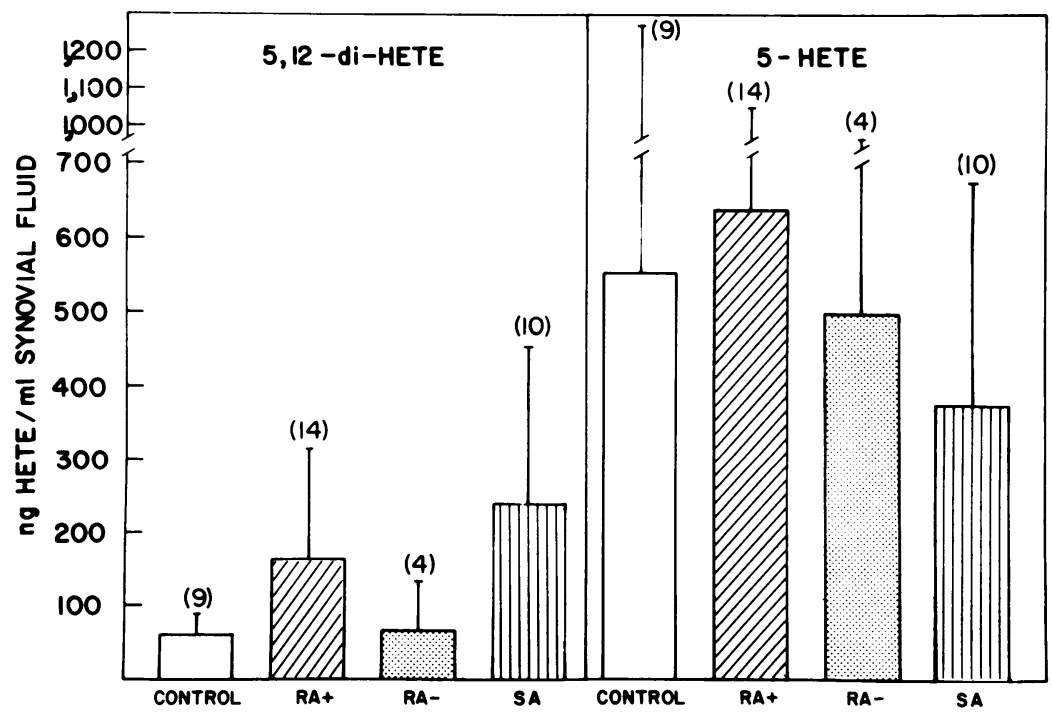

FIGURE 1 Concentrations of the predominant HETE in synovial fluid of arthritic patients. Each bar and bracket represents the mean \pm SD for the values of the number of patients shown in parentheses above the brackets. Statistical analyses employed Student's two-sample $t$ test with the Welch approximation. 


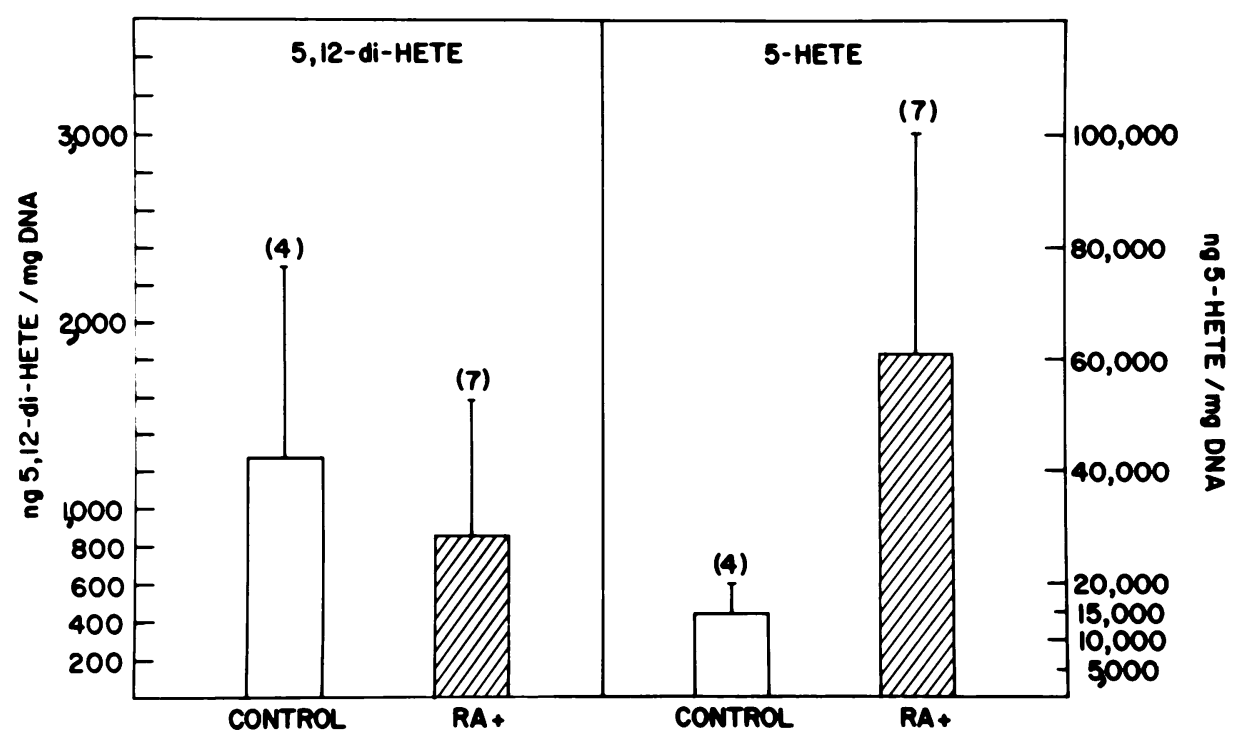

FIGURE 2 Levels of predominant HETE in synovial tissues of arthritic patients. The meaning of each bar and bracket and the statistical analyses are as in Fig. 1.

The effect of a single intra-articular injection of $40 \mathrm{mg}$ of 5-methyl prednisolone acetate was examined in six $\mathrm{RA}+$ patients. Before the injection of corticosteroid, the concentrations of $152 \pm 157 \mathrm{ng}( \pm \mathrm{SD})$ 5,12-di$\mathrm{HETE} / \mathrm{ml}$ and $542 \pm 182 \mathrm{ng} 5$-HETE $/ \mathrm{ml}$ were similar to those for other RA+ patients (Fig. 1). $3 \mathrm{~d}$ to $2 \mathrm{wk}$ after the intra-articular injection, the concentration of 5,12 -di-HETE had been reduced significantly to $67 \pm 40$ $\mathrm{ng} / \mathrm{ml}(p<0.05)$, whereas the concentration of 5-HETE was $526 \pm 431 \mathrm{ng} / \mathrm{ml}$ and had not been altered by the corticosteroid.

\section{DISCUSSION}

That lipoxygenase products of arachidonic acid are capable of eliciting inflammatory cellular infiltrates in vivo was demonstrated initially by the rapid influx of eosinophils and the later accumulation of neutrophils in response to the intraperitoneal instillation of microgram quantities of 12-L-HETE in guinea pigs (15). The possibility that lipoxygenase products are the predominant stimuli of the cellular component of some inflammatory reactions in vivo was suggested by the capacity of the lipoxygenase inhibitor BW755C to suppress the infiltration of PMN leukocytes evoked in the rat paw by the injection of carrageenin, while the cyclooxygenase inhibitor indomethacin prevented the alterations in vascular permeability, but not the leukocyte response (16). The present data indicate that the synovial levels of the lipoxygenase-derived PMN leukocyte chemotactic factors 5,12-di-HETE or leukotriene $B_{4}$ and 5-HETE are elevated in relation to active synovitis and compared with the levels in synovia of subjects with noninflammatory arthropathies
(Figs. $1 \& 2$ ). Leukotriene $\mathrm{B}_{4}$ is chemotactic for neutrophils and eosinophils in vitro at a concentration as low as $3 \mathrm{ng} / \mathrm{ml}$ and evokes a maximal chemotactic response at $30 \mathrm{ng} / \mathrm{ml}$, compared with $1,000 \mathrm{ng} / \mathrm{ml}$ for 5-HETE and 10,000-20,000 ng/ml for 11-HETE and 12-HETE (10). Thus, the concentration of both leukotriene $\mathrm{B}_{4}$ and 5-HETE in synovial fluid may be sufficient to contribute to the local inflammatory reaction (Fig. 1). The reduction in the synovial fluid level of leukotriene $B_{4}$ that followed the intra-articular injection of a corticosteroid in patients with rheumatoid arthritis is similar to the suppression of cutaneous levels of 12-HETE after topical corticosteroid therapy in psoriasis (17). The reduction in the synovial fluid leukocyte count and the transformation of the residual population from PMN to mononuclear leukocytes after the intra-articular injection of a corticosteroid (18) thus may be mediated in part by the suppression of the generation of lipoxygenase products of arachidonic acid, which are preferentially chemotactic for PMN leukocytes.

\section{ACKNOWLEDGMENT}

This work was supported in part by grant AI-07722 from the National Institutes of Health.

\section{REFERENCES}

1. Roberts, L. J., II, R. A. Lewis, J. A. Oates, and K. F. Austen. 1979. Prostaglandin, thromboxane, and 12-hydroxy$5,8,10,14$-eicosatetraenoic acid production by ionophorestimulated rat serosal mast cells. Biochim. Biophys. Acta. 575: 185-192.

2. Borgeat, P., and B. Samuelsson. 1979. Arachidonic acid 
metabolism in polymorphonuclear leukocytes: effects of ionophore A23187. Proc. Natl. Acad. Sci. U. S. A. 76: 2148-2152.

3. Goetzl, E. J., and F. F. Sun. 1979. Generation of unique mono-hydroxy-eicosatetraenoic acids from arachidonic acid by human neutrophils. J. Exp. Med. 150: 406-411.

4. Valone, F. H., M. Franklin, F. F. Sun, and E. J. Goetzl. 1980. Alveolar macrophage lipoxygenase products of arachidonic acid. Isolation and recognition as the predominant constituents of the neutrophil chemotactic activity elaborated by alveolar macrophages. Cell. Immunol. 54: 390-401.

5. Borgeat, P., and B. Samuelsson. 1979. Arachidonic acid metabolism in polymorphonuclear leukocytes: unstable intermediate in formation of dihydroxy acids. Proc. Natl. Acad. Sci. U. S. A. 76: 3213-3217.

6. Murphy, R. C., S. Hammarstrom, and B. Samuelsson. 1979. Leukotriene $C$ : a slow reacting substance from murine mastocytoma cells. Proc. Natl. Acad. Sci. U. S. A. 76: 4275-4270.

7. Parker, C. W., M. M. Huber, M. K. Hoffman, and S. F. Falkenhein. 1979. Characterization of the two major species of slow reacting substance from rat basophilic leukemia cells as glutathionyl thioethers of eicosatetraenoic acids oxygenated at the 5-position. Evidence that peroxy groups are present and important for spasmogenic activity. Prostaglandins. 18: 673-679.

8. Goetzl, E. J., P. F. Weller, and F. F. Sun. 1980. The regulation of human eosinophil function by endogenous mono-hydroxy-eicosatetraenoic acids (HETEs). J. Immunol. 124: 926-933.

9. Goetzl, E. J., A. R. Brash, A. I. Tauber, J. A. Oates, and W. C. Hubbard. 1980. Modulation of human neutrophil function by mono-hydroxy-eicosatetraenoic acids. Immunology. 39: 491-501.

10. Goetzl, E. J., and W. C. Pickett. 1980. The human PMN leukocyte chemotactic activity of complex hydroxy-eicosatetraenoic acids (HETEs). J. Immunol. 125: 1789-1791.

11. Hammarstrom, S., M. Hamberg, B. Samuelsson, E. A. Duell, M. Stawiski, and J. J. Voorhees. 1975. Increased concentrations of nonesterified arachidonic acid, $12 \mathrm{~L}-$ hydroxy-5,8,10,14-eicosatetraenoic acid, prostaglandin $\mathrm{E}_{2}$ and prostaglandin $F_{2 \alpha}$ in epidermis of psoriasis. Proc. Natl. Acad. Sci. U. S. A. 72: 5130-5134.

12. Goetzl, E. J. 1980. A role for endogenous mono-hydroxyeicosatetraenoic acids (HETEs) in the regulation of human neutrophil migration. Immunology. 40: 709-722.

13. Kissane, J. M., and E. Robins. 1958. The fluorometric measurement of deoxyribonucleic acid in animal tissues with special reference to the central nervous system. $J$. Biol. Chem. 233: 184-188.

14. Lowry, O. H., N. J. Rosebrough, A. L. Farr, and R. J. Randall. 1951. Protein measurement with the Folin phenol reagent. J. Biol. Chem. 193: 265-275.

15. Goetzl, E. J., F. H. Valone, V. N. Reinhold, and R. R. Gorman. 1979. Specific inhibition of the polymorphonuclear leukocyte chemotactic response to hydroxy-fatty acid metabolites of arachidonic acid by methyl ester derivatives. J. Clin. Invest. 63: 1181-1186.

16. Higgs, G. A., S. Moncada, and J. R. Vane. 1979. The role of arachidonic acid metabolites in inflammation. In Advances in Inflammation Research. G. Weissman, B. Samuelsson, and R. Paoletti, editors. Raven Press, New York. Vol. 1. 413-418.

17. Hammarstrom, S., M. Hamberg, E. A. Duell, M. A. Stawiski, T. F. Anderson, and J. J. Voorhees. 1977. Glucocorticoid in inflammatory proliferative skin disease reduces arachidonic and hydroxyeicosatetraenoic acids. Science (Wash. D. C.). 205: 994-995.

18. Goetzl, E. J., N. E. Bianco, J. S. Alpert, C. B. Sledge, and P. H. Schur. 1974. Effects of intra-articular corticosteroids in vivo on synovial fluid variables in rheumatoid synovitis. Ann. Rheum. Dis. 33: 420-424. 